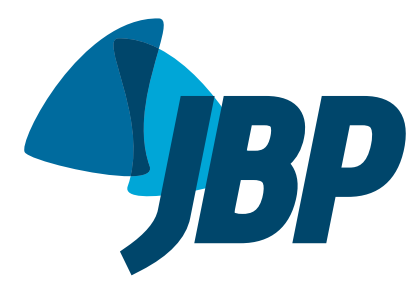

1. Research Institute of Phthisiopulmonology, I.M. Sechenov First Moscow State Medical University, Moscow, Russia

2. Medical Center, Central Bank of Russian Federation, Moscow, Russia.

Submitted: 2 March 2016.

Accepted: 15 August 2016.

Study carried out in the Laboratory of

Pulmonary Physiology, Research Institute of Phthisiopulmonology, I.M. Sechenov First Moscow State Medical University, Moscow, Russia.

\title{
Impaired pulmonary function after treatment for tuberculosis: the end of the disease?
}

\author{
Mikhail Ivanovich Chushkin ${ }^{1,2}$, Oleg Nikolayevich Ots ${ }^{1}$
}

\begin{abstract}
Objective: To evaluate the prevalence of pulmonary function abnormalities and to investigate the factors affecting lung function in patients treated for pulmonary tuberculosis. Methods: A total of 214 consecutive patients (132 men and 82 women; 2082 years of age), treated for pulmonary tuberculosis and followed at a local dispensary, underwent spirometry and plethysmography at least one year after treatment. Results: Pulmonary impairment was present in 102 (47.7\%) of the 214 patients evaluated. The most common functional alteration was obstructive lung disease (seen in $34.6 \%$ ). Of the 214 patients, 60 (28.0\%) showed reduced pulmonary function ( $F E V_{1}$ below the lower limit of normal). Risk factors for reduced pulmonary function were having had culturepositive pulmonary tuberculosis in the past, being over 50 years of age, having recurrent tuberculosis, and having a lower level of education. Conclusions: Nearly half of all tuberculosis patients evolve to impaired pulmonary function. That underscores the need for pulmonary function testing after the end of treatment.
\end{abstract}

Keywords: Respiratory function tests; Tuberculosis, pulmonary; Spirometry; Lung diseases, obstructive.

\section{INTRODUCTION}

It has been estimated that approximately one third of the world's population is latently infected with Mycobacterium tuberculosis. ${ }^{(1)}$ In 2011, there were an estimated 8.7 million new cases of tuberculosis worldwide, equivalent to 125 cases per 100,000 population. ${ }^{(1)}$

In patients infected with M. tuberculosis, whether treated or untreated, a variety of pulmonary and extrapulmonary sequelae and complications can occur categorized as follows: parenchymal lesions, which include tuberculoma, thin-walled cavities, scarring, and end-stage lung destruction; or airway lesions, which include bronchiectasis, tracheobronchial stenosis, and broncholithiasis. (2) Structural changes lead to obstructive, restrictive, or mixed patterns of impaired pulmonary function. Studies in patients with pulmonary tuberculosis (PTB) have demonstrated that $33.3-94.0 \%$ of such patients develop impaired pulmonary function. ${ }^{(3)}$ Although it is unknown how many PTB survivors are living today, when the incidence of tuberculosis and the success of therapy are considered, the number of PTB survivors appears to be substantial and increasing. ${ }^{(4)}$ There have been few studies on the topic of impaired pulmonary function in PTB survivors, and most such studies have involved highly selected populations. The patients in those populations do not fully represent the populations affected by tuberculosis. ${ }^{(4-8)}$ Little is known about the prevalence of a restrictive pattern after PTB. Although the evaluation of true restriction requires the measurement of TLC, the studies cited above used only spirometry. Most of those studies were conducted in countries where the incidence of tuberculosis is low; the prevalence and type of pulmonary impairment might differ in countries where that incidence is high.

The objectives of this study were to evaluate the incidence and extent of pulmonary function abnormalities in patients previously treated for PTB. We also studied the factors affecting pulmonary function in such patients.

\section{METHODS}

This study was carried out in the Department of Pulmonary Physiology, Research Institute of Phthisiopulmonology, I.M. Sechenov First Moscow State Medical University, in Moscow, Russia. The study was approved by the Ethical Review Board of the Association of Medical and Pharmaceutical Universities. All participants gave written informed consent, and confidentiality was ensured.

In Russia, the primary component of the tuberculosis control system is the regional dispensary, which provides services to patients with active disease and to those considered at risk of contracting or developing the disease. The patients are followed for some time after treatment. ${ }^{(9)}$

In the 2003-2007 period, a total of 757 PTB patients (between 20 and 90 years of age) were treated and followed at the local tuberculosis dispensary. All 757 patients were subsequently recruited by telephone. Letters were sent if no telephone contact was made. Of the 757 eligible patients, 214 agreed to take part in the study.

From April 2005 to December 2013, patients previously treated for PTB underwent pulmonary function tests

Correspondence to:

Mikhail Chushkin. Laboratory of Pulmonary Physiology, Research Institute of Phthisiopulmonology, Sechenov First Moscow State Medical University, 4 bld Dostoevsky,

127994, Moscow, Russia.

Tel.: 7 915 485-76-50. E-mail: mchushkin@yandex.ru

Financial support: None. 
(PFTs) in the Department of Pulmonary Physiology. All 214 of the patients had been treated successfully, and the PFTs were performed at least one year after the end of treatment. At the time of the PFTs, none of the patients showed any signs of active PTB.

All of the participating patients underwent spirometry and plethysmography, as well as completing a questionnaire designed to collect data related to demographic characteristics, smoking, medical history, and other aspects. The PFTs were performed by experienced technicians, in accordance with the recommendations of the American Thoracic Society/ European Respiratory Society, ${ }^{(10,11)}$ with a combination spirometer/plethysmograph (MasterScreen Body; Jaeger, Würzburg, Germany). Of the 214 patients, 69 had physician-diagnosed chronic lung disease. All 214 patients were urban dwellers without a personal history of exposure to biomass smoke. Those with a history of intermittent bronchodilator use received their usual treatment 30 min prior to testing. European Community for Coal and Steel equations for spirometry and lung volumes were applied.(12) Airway obstruction was defined as an $\mathrm{FEV}_{1} / \mathrm{VC}$ ratio below the lower limit of normal (LLN) and a TLC above or equal to the LLN; a restrictive pattern was defined as a TLC below the LLN and an $\mathrm{FEV}_{1} / \mathrm{VC}$ ratio above or equal to the LLN; a mixed pattern was defined as an $\mathrm{FEV}_{1} / \mathrm{VC}$ ratio and TLC both below the LLN ${ }^{(13)}$; and a nonspecific pattern was defined as a TLC above or equal to the LLN, an $\mathrm{FEV}_{1} / \mathrm{VC}$ ratio above or equal to the LLN, and an $\mathrm{FEV}_{1}$ or FVC below the LLN.(14) We defined reduced pulmonary function as an FEV 1 below the LLN.

For quantitative variables, the differences between groups were assessed by the Mann-Whitney test or the Kruskal-Wallis test. For categorical variables, the groups were compared by the chi-square test. The predictive value of age for the presence of pulmonary impairment was evaluated by determining the area under the ROC curve. Accuracy was calculated for the best cut-off value, defined as that with the highest sum of sensitivity and specificity. Logistic regression was used in order to identify factors associated with impaired pulmonary function. Values of $p<0.05$ were considered statistically significant.

\section{RESULTS}

Table 1 summarizes the main characteristics of the 214 subjects included in the analysis. There were 132 men and 82 women, with a mean age of 51.1 years (range, 20-82 years). Of the 214 subjects, 105 had previously been culture-positive and 90 had previously been culture-negative. For the remaining 19 subjects, the culture results were unknown.

The PFT results were normal in 112 (52.4\%) of the 214 patients (Table 2). Pulmonary impairment was identified in $102(47.7 \%)$ of the patients, the pattern being obstructive in $74(34.6 \%)$, restrictive in 18 $(8.4 \%)$, mixed in $8(3.7 \%)$, and nonspecific in $2(0.9 \%)$.
Of the 214 patients, 60 (28.0\%) had an FEV , below the LLN. Using the American Thoracic Society/European Respiratory Society criteria, ${ }^{(13)}$ we classified the degree of abnormality as mild in $6(2.8 \%)$, moderate in 34 $(15.9 \%)$, and severe in $20(9.3 \%)$, as detailed in Table 2. Clinically significant pulmonary impairment, defined as an $\mathrm{FEV}_{1}<60 \%$ of the predicted value, ${ }^{(15)}$ was identified in $35(14.5 \%)$ of the subjects, being attributed to an obstructive pattern in 22 (10.3\%), a restrictive pattern in $5(2.3 \%)$, and a mixed pattern in $8(3.7 \%)$. Of those 35 patients, 13 had not been diagnosed with a chronic pulmonary disease.

According to the ROC curve analysis for reduced pulmonary function, when the age of 50 was chosen as the cut-off value, the sensitivity was $73.3 \%$ and the specificity was $59.1 \%$. The area under the curve was 0.67 (95\% CI: 0.59-0.76; $\mathrm{p}=0.002$ ).

As can be seen in Table 3, the prevalence of any pulmonary impairment in the patients $\geq 50$ years of age was $60.6 \%$ (range, 56.3-63.3\%, depending on the specific age bracket), compared with $34.3 \%$ (range, $7.1-42.4 \%$, depending on the specific age bracket) in

Table 1. Characteristics of the patients in the study sample $(n=214)$.

\begin{tabular}{|c|c|c|}
\hline Characteristic & Value & Range \\
\hline \multicolumn{3}{|l|}{ Gender $^{\mathrm{a}}$} \\
\hline Male & $132(61.7)$ & \\
\hline Female & $82(38.3)$ & \\
\hline Age, years ${ }^{b}$ & $51.1(14.2)$ & $20-82$ \\
\hline$<40^{\mathrm{a}}$ & $46(21.5)$ & \\
\hline $40-49$ & $59(27.6)$ & \\
\hline $50-59$ & $49(22.9)$ & \\
\hline $60-69$ & $32(15.0)$ & \\
\hline$\geq 70$ & $28(13.1)$ & \\
\hline $\mathrm{BMI}, \mathrm{kg} / \mathrm{m}^{2 \mathrm{~b}}$ & $23.9(4.4)$ & $14.8-41.5$ \\
\hline \multicolumn{3}{|l|}{ Education $^{\mathrm{a}, \mathrm{c}}$} \\
\hline College-educated & $66(31.4)$ & \\
\hline High school only & $144(68.6)$ & \\
\hline \multicolumn{3}{|l|}{ Smoking status ${ }^{\mathrm{a}}$} \\
\hline Current smoker & $112(52.3)$ & \\
\hline Former smoker & $26(12.2)$ & \\
\hline Never-smoker & $76(35.5)$ & \\
\hline \multicolumn{3}{|l|}{ Pulmonary function ${ }^{\mathrm{b}}$} \\
\hline FVC, $\%$ of predicted & $99.7(21.4)$ & $31-143$ \\
\hline $\mathrm{FEV}_{1}, \%$ of predicted & $87.1(25.0)$ & $23-139$ \\
\hline $\mathrm{FEV}_{1} / \mathrm{VC}$ ratio & $70.3(13.5)$ & 28.7-97 \\
\hline PEF, \% of predicted & $81.9(28.7)$ & $21-151$ \\
\hline MMEF, $\%$ of predicted & $61.9(33.3)$ & $5.4-161$ \\
\hline TLC, $\%$ of predicted & $99.9(17.8)$ & $38-136$ \\
\hline FRC, $\%$ of predicted & $111.4(27.9)$ & $45-191$ \\
\hline $\mathrm{RV}, \%$ of predicted & $103.9(30.2)$ & $34-253$ \\
\hline IC, $\%$ of predicted & $91.2(24.2)$ & $27-165$ \\
\hline $\mathrm{RV} / \mathrm{TLC}$ ratio & $34.7(10.0)$ & $16-74$ \\
\hline
\end{tabular}

BMI: body mass index; MMEF: maximal midexpiratory flow; FRC: functional residual capacity; and IC: inspiratory capacity. ${ }^{\text {Values expressed as } \mathrm{n}}$

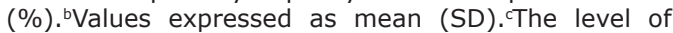
education was unknown in 4 patients. 
the patients $<50$ years of age $(p<0.001$; chi-square test). In the patients $\geq 50$ of age, the prevalence of reduced pulmonary function was $40.4 \%$ (range, $37.5-42.9 \%$, depending on the specific age bracket), whereas it was $15.2 \%$ (range, $0.0-20.3 \%$, depending on the specific age bracket) in the patients $<50$ years of age, and the difference between the two groups was statistically significant ( $p<0.001$; chi-square test).

Table 4 shows the pulmonary function parameters, stratified by culture results, number of episodes of PTB, and smoking history. The values for FVC, FEV ${ }_{1}$ and the $\mathrm{FEV}_{1} / \mathrm{VC}$ ratio were significantly lower in the patients who had previously had culture-positive PTB than in those who had previously had culture-negative PTB ( $p<0.05$ for all; Mann-Whitney test). There were 188 patients who had had only one episode of PTB and 26 who had had two or more episodes. Values for $\mathrm{FEV}_{1}$ and the $\mathrm{FEV}_{1} / \mathrm{VC}$ ratio were significantly lower in the patients who had had two or more episodes of PTB than in those who had had only one. More than $60 \%$ of the subjects evaluated in our study had a history of smoking. The prevalence of airway obstruction in ever-smokers (smokers and former smokers, collectively) and never-smokers was $47.1 \%$ and $22.4 \%$, respectively ( $p<0.001$; chi-square test). The prevalence of reduced pulmonary function in ever-smokers and in never-smokers was $31.2 \%$ and

Table 2. Type and severity of pulmonary impairment in patients previously treated for pulmonary tuberculosis. ${ }^{a}$

\begin{tabular}{|c|c|}
\hline Variable & $(n=214)$ \\
\hline \multicolumn{2}{|l|}{ Type of impairment } \\
\hline Normal & $112(52.4)$ \\
\hline Obstructive pattern $\left(\mathrm{FEV}_{1} / \mathrm{VC}\right.$ ratio $<\mathrm{LLN}$ and $\left.\mathrm{TLC} \geq \mathrm{LLN}\right)$ & $74(34.6)$ \\
\hline Restrictive pattern (TLC < LLN and FEV $/$ VC ratio $\geq$ LLN) & $18(8.4)$ \\
\hline Mixed pattern ( $\mathrm{TLC}$ and $\mathrm{FEV}_{1} / \mathrm{VC}$ ratio < LLN) & $8(3.7)$ \\
\hline Nonspecific pattern (TLC $\geq$ LLN, $\mathrm{FEV}_{1} / \mathrm{VC}$ ratio $\geq \mathrm{LLN}$, and $\mathrm{FEV}_{1}$ or FVC $<\mathrm{LLN}$ ) & $2(0.9)$ \\
\hline Reduced pulmonary function $\left(\mathrm{FEV}_{1}<\mathrm{LLN}\right)$ & $60(28)$ \\
\hline \multicolumn{2}{|l|}{$\mathrm{FEV}_{1}$} \\
\hline$<35 \%$ of predicted & $9(4.2)$ \\
\hline $35-49 \%$ of predicted & $11(5.1)$ \\
\hline $50-59 \%$ of predicted & $15(7.0)$ \\
\hline $60-69 \%$ of predicted & $19(8.9)$ \\
\hline$\geq 70 \%$ of predicted and $<$ LLN & $6(2.8)$ \\
\hline$\geq$ LLN & $154(72)$ \\
\hline
\end{tabular}

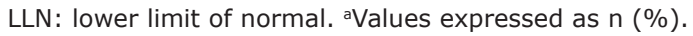

Table 3. Relationship between age and pulmonary impairment in patients previously treated for pulmonary tuberculosis. ${ }^{a}$

\begin{tabular}{|c|c|c|c|c|c|c|c|}
\hline \multirow[t]{2}{*}{ Age, years } & \multirow[t]{2}{*}{ Gender } & \multirow[t]{2}{*}{$\mathbf{n}$} & \multirow{2}{*}{$\begin{array}{c}\text { Any } \\
\text { impairment }\end{array}$} & \multicolumn{3}{|c|}{ Pattern of impairment ${ }^{b, c}$} & \multirow{2}{*}{$\begin{array}{l}\text { Reduced pulmonary } \\
\text { function }{ }^{d}\end{array}$} \\
\hline & & & & Obstructive & Restrictive & Mixed & \\
\hline & Male & 7 & & & & & \\
\hline \multirow[t]{3}{*}{$20-29$} & Female & 7 & $1(7.1)$ & $1(7.1)$ & $0(0)$ & $0(0)$ & $0(0)$ \\
\hline & Total & 14 & & & & & \\
\hline & Male & 22 & & & & & \\
\hline \multirow[t]{3}{*}{$30-39$} & Female & 10 & $10(31.3)$ & $6(18.8)$ & $2(6.3)$ & $1(3.1)$ & $4(12.5)$ \\
\hline & Total & 32 & & & & & \\
\hline & Male & 39 & & & & & \\
\hline \multirow[t]{3}{*}{$40-49$} & Female & 20 & $25(42.4)$ & $24(40.7)$ & $1(1.7)$ & $0(0)$ & $12(20.3)$ \\
\hline & Total & 59 & & & & & \\
\hline & Male & 36 & & & & & \\
\hline \multirow[t]{3}{*}{$50-59$} & Female & 13 & $31(63.3)$ & $21(42.9)$ & $7(14.3)$ & $3(6.1)$ & $21(42.9)$ \\
\hline & Total & 49 & & & & & \\
\hline & Male & 18 & & & & & \\
\hline \multirow[t]{3}{*}{$60-69$} & Female & 14 & $18(56.3)$ & $13(40.6)$ & $2(6.25)$ & $2(6.3)$ & $12(37.5)$ \\
\hline & Total & 32 & & & & & \\
\hline & Male & 10 & & & & & \\
\hline \multirow[t]{2}{*}{$\geq 70$} & Female & 18 & $17(60.7)$ & $9(32.1)$ & $6(21.43)$ & $2(7.1)$ & $11(39.3)$ \\
\hline & Total & 28 & & & & & \\
\hline
\end{tabular}

LLN $=$ lower limit of normal. ${ }^{a}$ Values are given as $\mathrm{n}(\%) .{ }^{\mathrm{b}} \mathrm{An}$ obstructive pattern was defined as an $\mathrm{FEV}_{1} / \mathrm{VC}$ ratio $<$ the lower limit of normal (LLN) and a TLC $\geq$ LLN; a restrictive pattern was defined as a TLC $<$ LLN and an FEV, /VC ratio $\geq L L N$; a mixed pattern was defined as an $\mathrm{FEV}_{1} / \mathrm{VC}$ ratio $<$ LLN and a TLC < LLN; and a nonspecific pattern

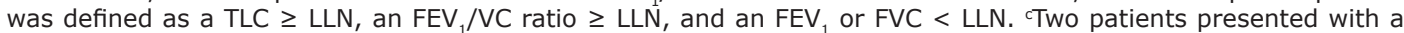

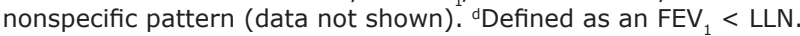


$22.4 \%$, respectively ( $p=0.226$; chi-square test). In the smokers, former smokers, and never-smokers, the mean $\mathrm{FEV}_{1}$ (as a percentage of the predicted value) was $87.0 \pm 22.7 \%, 80.0 \pm 27.5 \%$, and 89.7 $\pm 27.1 \%$, respectively ( $p=0.201$; Kruskal-Wallis test). We found that smoking had no influence on the prevalence of reduced pulmonary function. Only the $\mathrm{FEV}_{1} / \mathrm{VC}$ ratio was lower in ever-smokers than in never-smokers (Table 4).

The risk factors for reduced pulmonary function were having previously had culture-positive PTB, being over 50 years of age, having a low level of education, and having experienced recurrence of tuberculosis. The prevalence of reduced pulmonary function was lower in college-educated patients than in those who had only a high school education ( $18.2 \%$ vs. $31.9 \%)$, and the difference was statistically significant $(p<0.05$; chi-square test). We found that neither gender nor smoking status had any influence on the prevalence of reduced pulmonary function (Table 5 ).

\section{DISCUSSION}

A study conducted in the United States showed that $59 \%$ of patients treated for tuberculosis subsequently had abnormal pulmonary function. ${ }^{(4)}$ In that study, more than half of the patients treated for PTB evolved to significantly impaired pulmonary function. These data suggest that impaired pulmonary function after PTB is a major cause of chronic lung disease. ${ }^{(4)}$ The authors of that study found that $44 \%$ of the patients developed restrictive impairment, ${ }^{(4)}$ compared with the $6.6 \%$ reported for the general population. ${ }^{(16)}$ We also found that the prevalence of restrictive impairment was higher among the patients evaluated in our study than in the general population.

In the present study, approximately half of all PTB patients treated at the local tuberculosis dispensary suffered from impaired pulmonary function, a prevalence much higher than that observed in the general population. In addition to the risk factors for reduced pulmonary function identified in the present studyhaving previously had culture-positive PTB, being over 50 years of age, having a low level of education, and having experienced recurrence of tuberculosis-potential risk factors for impaired pulmonary function in patients previously treated for PTB include extensive disease before treatment, prolonged treatment duration, and poor radiographic improvement after treatment. ${ }^{(17)}$

We can presume that recurrent PTB is an important risk factor for pulmonary impairment and that patients with risk factors for recurrence require close monitoring and appropriate treatment in order to prevent future

Table 4. Conditional analysis of pulmonary impairment after treatment for pulmonary tuberculosis.

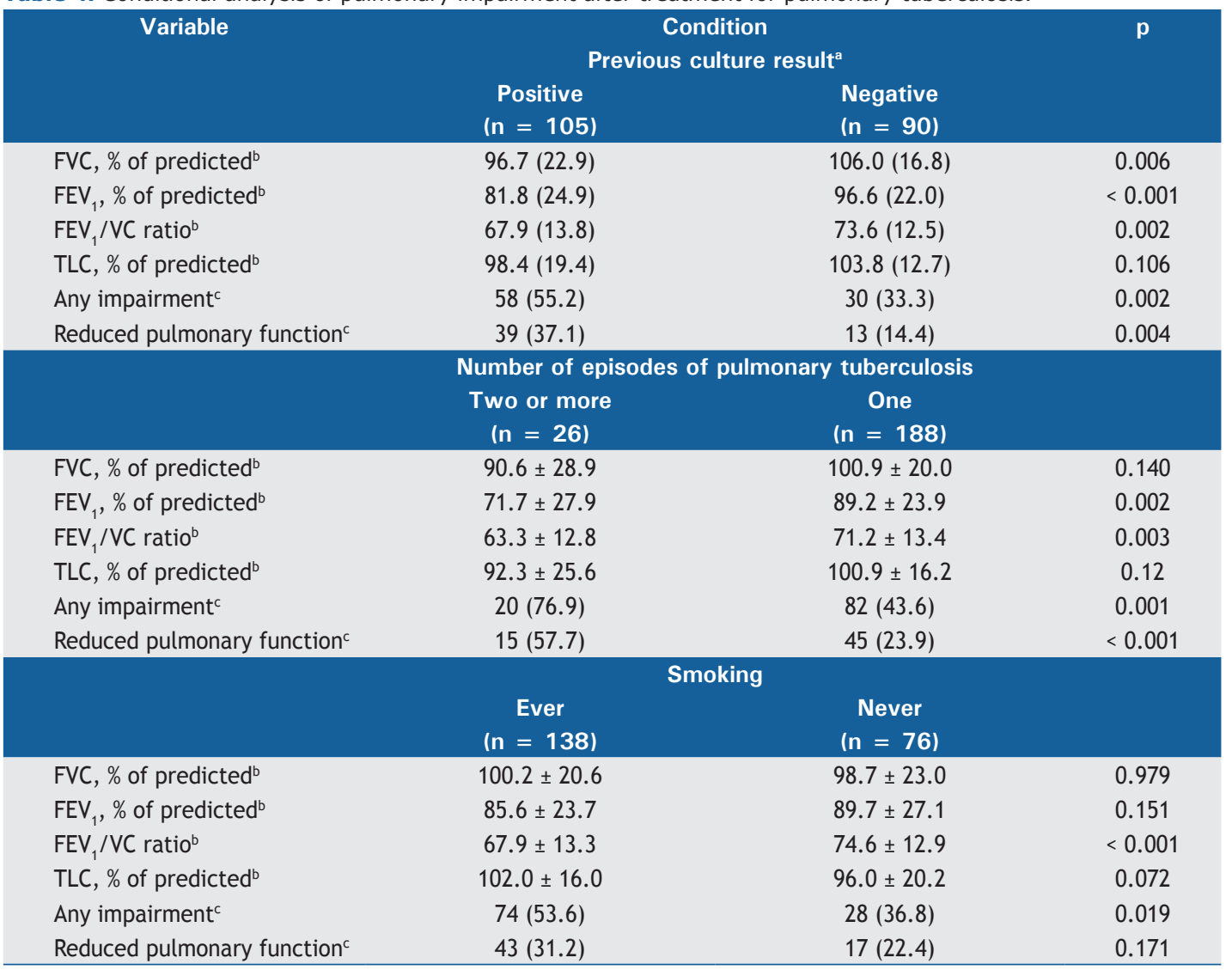

a Determined for only 195 of the 214 patients in the sample. ${ }^{b}$ Values expressed as mean (SD). ${ }^{\text {Values expressed }}$ as $\mathrm{n}(\%)$. ${ }^{\mathrm{d}}$ Defined as an $\mathrm{FEV}_{1}<\mathrm{LLN}$. 
Table 5. Risk factors for reduced pulmonary function in patients previously treated for pulmonary tuberculosis. ${ }^{\text {a }}$

\begin{tabular}{lccc}
\multicolumn{1}{c}{ Risk factor } & OR & $\mathbf{9 5 \%} \mathbf{C l}$ & p \\
Recurrence of tuberculosis: yes vs. no. & 4.33 & $1.86-10.11$ & $<0.001$ \\
Age: $<50$ years vs. $\geq 50$ years & 3.77 & $1.95-7.25$ & $<0.001$ \\
Positive culture in the past ${ }^{\text {b: }}$ yes vs. no & 3.5 & $1.72-7.11$ & $<0.001$ \\
Education: high school only vs. college education & 2.11 & $1.03-4.33$ & 0.04 \\
Smoking: ever vs. never & 1.57 & $0.82-3.0$ & 0.17 \\
Gender: male vs. female & 1.49 & $0.79-2.81$ & 0.21 \\
\hline
\end{tabular}

aDefined as an $\mathrm{FEV}_{1}$ below the lower limit of normal. betermined for only 195 of the 214 patients in the sample.

episodes of PTB. Hnizdo et al.(5) showed that the mean decrease in $\mathrm{FEV}_{1}$ was $180 \mathrm{~mL}$ in patients who had experienced only one episode of PTB, $362 \mathrm{~mL}$ in those who had experienced two episodes, and $462 \mathrm{~mL}$ in those who had experienced three episodes.

The prevalence of reduced pulmonary function was significantly lower in college-educated patients than in those who had only a high school education; $18.2 \%$ and $31.9 \%$, respectively ( $p<0.05$; chi-square test). As a rule, college-educated patients have a better socioeconomic status, better nutrition, less occupational risk and a more conscientious attitude toward treatment than do those without a college education. ${ }^{(18)}$ The magnitude of the effect of socioeconomic status has been reported to be $200-300 \mathrm{~mL}$ of FEV ${ }_{1}{ }^{(18)}$

Although smoking has been established as a major risk factor for COPD, an estimated $25-45 \%$ of COPD patients are never-smokers and emerging evidence suggests that other risk factors are important. ${ }^{(18)} \mathrm{A}$ recent meta-analysis showed that smoking is a risk factor for tuberculosis. However, it is not clear whether smoking can increase the mortality risk in individuals who already have active tuberculosis. ${ }^{(19)}$ The impact of smoking on pulmonary impairment in patients who have been treated for PTB is also unknown. Previous studies have produced inconsistent results. $(4,6,8,17)$ In our study, despite the fact that the prevalence of obstruction was significantly higher in ever-smokers than in never-smokers, we found that smoking had no influence on the prevalence of reduced pulmonary function. The explanation for that finding remains uncertain. Chung et al. ${ }^{(17)}$ found that a history of PTB was a stronger determinant of impaired pulmonary function than was smoking. Those authors also suggested that post-tuberculosis pulmonary inflammation can mask a smoking-related decline in pulmonary function. ${ }^{(17)}$

The overall prevalence of pulmonary impairment is much higher in patients treated for PTB than in the general population. In our patient sample, obstructive impairment was the most common pattern, followed by restrictive, mixed, and nonspecific impairment. These results are in accordance with those of other studies in the literature ${ }^{(4)}$ suggesting that pulmonary impairment after tuberculosis could be an underestimated cause of chronic lung disease, especially in countries where the tuberculosis burden is high. Reduced $\mathrm{FEV}_{1}$ has been shown to be an independent predictor of all-cause and respiratory disease-related mortality. ${ }^{(20)}$
Inghammar et al. ${ }^{(21)}$ showed that impaired pulmonary function was associated with an increased risk of active tuberculosis. However, if decreased lung function is a risk factor for the development of tuberculosis, it is also possible that pulmonary impairment is a risk factor for its recurrence. Further studies are needed in order to determine whether impaired pulmonary function can be viewed as a risk factor and to devise targeted measures to prevent the recurrence of tuberculosis.

The presence of symptoms is not a sensitive and specific indicator of airway limitation, and the use of a symptom questionnaire appears to be an ineffective means of identifying pulmonary impairment. ${ }^{(22)}$ However, evidence suggests that pulmonary function testing is not used consistently. Even in developed countries, less than half of all patients newly diagnosed with chronic pulmonary diseases receive pulmonary function testing near the time of diagnosis. ${ }^{(23)}$ Using a mechanical peak expiratory flow meter or a pocket spirometer as a screening tool can reduce the number of diagnostic PFTs required. ${ }^{(24)}$ Although it has not been established that PFTs can predict recurrence, their use can probably help select a group of patients at higher risk of recurrence who require longer follow-up and prevention measures. This supports the idea that patients previously treated for PTB should undergo pulmonary function testing. However, pulmonary function testing has yet to be included in the guidelines for the treatment of tuberculosis. ${ }^{(25)}$ We suppose that using $\mathrm{FEV}_{1}<80 \%$ of the predicted value (rather than $\mathrm{FEV}_{1}$ below the LLN) as a cut-off value would serve as a reasonable strategy for patients under follow-up treatment, because of its simplicity and ease of use.

This study has some limitations. Very few of the patients received bronchodilators, and it is possible that bronchospasm is conducive to pulmonary impairment. In addition, the study was conducted at a single center, whereas a multicenter study could have produced results that would have been more robust. Furthermore, because of a lack of the pertinent data, we did not examine the influence that a history of cavitation had on pulmonary impairment. Tuberculosis treatment is much less effective in patients with cavitary disease than in those with noncavitary disease or culture-positive PTB, and the negative influence of cavitary disease on lung function could therefore be quite significant. (26)

In conclusion, we found that a history of PTB was a risk factor for pulmonary impairment. The eradication of the bacteria does not necessarily translate to the end 
of the illness. After tuberculosis treatment, more than $40 \%$ of patients can evolve to pulmonary impairment, mainly obstructive disorders. This underscores the need for pulmonary function testing in patients who have been treated for PTB.

\section{ACKNOWLEDGMENTS}

The authors thank Tatyana Radina, Vera Plotnikova, and Liubov Yashina for their help in preparing the manuscript.

\section{REFERENCES}

1. Glaziou P, Falzon D, Floyd K, Raviglione M. Global epidemiology of tuberculosis. Semin Respir Crit Care Med. 2013;34(1):3-16. https:// doi.org/10.1055/s-0032-1333467

2. Kim HY, Song KS, Goo JM, Lee JS, Lee KS, Lim TH. Thoracic sequelae and complications of tuberculosis. Radiographics. 2001; 21(4):839-58; discussion 859-60. https://doi.org/10.1148/ radiographics.21.4.g01j106839

3. Stepanian IE. Bronchial impotence in patients with pulmonary tuberculosis [Article in Russian]. Tuberk Biolezni Legkih. 2013;4(1): 6-11.

4. Pasipanodya JG, Miller TL, Vecino M, Munguia G, Garmon R, Bae S, et al. Pulmonary impairment after tuberculosis. Chest. 2007:131(6): 1817-24. https://doi.org/10.1378/chest.06-2949

5. Hnizdo $E$, Singh $T$, Churchyard $G$. Chronic pulmonary function impairment caused by initial and recurrent pulmonary tuberculosis following treatment. Thorax. 2000;55(1):32-8. https://doi.org/10.1136/ thorax.55.1.32

6. Lee SW, Kim YS, Kim DS, Oh YM, Lee SD. The risk of obstructive lung disease by previous pulmonary tuberculosis in a country with intermediate burden of tuberculosis. J Korean Med Sci. 2011;26(2):268-73. https://doi.org/10.3346/jkms.2011.26.2.268

7. Menezes AM, Hallal PC, Perez-Padilla R, Jardim JR, Muiño A, Lopez $M V$, et al. Tuberculosis and airflow obstruction: evidence from the PLATINO study in Latin America. Eur Respir J. 2007;30(6):1180-5. https://doi.org/10.1183/09031936.00083507

8. Willcox PA, Ferguson AD. Chronic obstructive airways disease following treated pulmonary tuberculosis. Respir Med. 1989;83(3):195-8. https://doi.org/10.1016/S0954-6111(89)80031-9

9. Perelman MI. Tuberculosis in Russia. Int $\mathrm{J}$ Tuberc Lung Dis 2000;4(12):1097-103.

10. Miller MR, Hankinson J, Brusasco V, Burgos F, Casaburi R, Coates A, et al; ATS/ERS Task Force. Standardisation of spirometry. Eur Respir J. 2005;26(2):319-38. https://doi.org/10.1183/09031936.05.0 0034805

11. Wanger J, Clausen JL, Coates A, Pedersen OF, Brusasco V, Burgos, et al. Standardisation of the measurement of lung volumes. Eur Respir J 2005; 26: 511-522. https://doi.org/10.1183/09031936.05.0 0035005

12. Quanjer $\mathrm{PH}$, Tammeling GJ, Cotes JE, Pedersen OF, Peslin R Yernault JC. Lung volumes and forced ventilatory flows. Work Group on Standardization of Respiratory Function Tests. European Community for Coal and Steel. Official position of the European Respiratory Society [Article in French]. Rev Mal Respir. 1994;11 Suppl 3:5-40.

13. Pellegrino R, Viegi G, Brusasco V, Crapo RO, Burgos F, Casaburi R et al. Interpretative strategies for lung function tests. Eur Respir J. 2005;26(5):948-68. https://doi.org/10.1183/09031936.05.00035205

14. Iyer VN, Schroeder DR, Parker KO, Hyatt RE, Scanlon PD. The nonspecific pulmonary function test: longitudinal follow-up and outcomes. Chest. 2011;139(4): 878-86. https://doi.org/10.1378/ chest.10-0804

15. Qaseem A, Wilt TJ, Weinberger SE, Hanania NA, Criner G, van der Molen $T$, et al. Diagnosis and management of stable chronic obstructive pulmonary disease: a clinical practice guideline update from the American College of Physicians, American College of Chest Physicians, American Thoracic Society, and European Respiratory Society. Ann Intern Med. 2011;155(3):179-91. https:// doi.org/10.7326/0003-4819-155-3-201108020-00008

16. Mannino DM, Ford ES, Redd SC. Obstructive and restrictive lung disease and functional limitation: data from the Third National Health and Nutrition Examination. J Intern Med. 2003;254(6):540-7. https:// doi.org/10.1111/j.1365-2796.2003.01211.x

17. Chung KP, Chen JY, Lee CH, Wu HD, Wang JY, Lee LN, et al. Trends and predictors of changes in pulmonary function after treatment for pulmonary tuberculosis. Clinics (Sao Paulo). 2011:66(4):549-56. https://doi.org/10.1590/S1807-59322011000400005

18. Salvi SS, Barnes PJ. Chronic obstructive pulmonary disease in nonsmokers. Lancet. 2009;374(9691):733-43. https://doi.org/10.1016/ S0140-6736(09)61303-9

19. Bates MN, Khalakdina A, Pai M, Chang L, Lessa F, Smith KR. Risk of tuberculosis from exposure to tobacco smoke: a systematic review and meta-analysis. Arch Intern Med. 2007:167(4):335-42. https://doi. org/10.1001/archinte.167.4.335

20. Young RP, Hopkins R, Eaton TE. Forced expiratory volume in one second: not just a lung function test but a marker of premature death from all causes. Eur Respir J. 2007;30(4):616-22. https://doi. org/10.1183/09031936.00021707

21. Inghammar $M$, Löfdahl CG, Winqvist $N$, Ljungberg $B$, Egesten $A$ Engström G. Impaired pulmonary function and the risk of tuberculosis: a population-based cohort study. Eur Respir J. 2011;37(5):1285-7. https://doi.org/10.1183/09031936.00091110

22. Buffels J, Degryse J, Heyrman J, Decramer M; DIDASCO Study Office spirometry significantly improves early detection of COPD in general practice: the DIDASCO Study. Chest. 2004;125(4):1394-9. https://doi.org/10.1378/chest.125.4.1394

23. Lee TA, Bartle B, Weiss KB. Spirometry use in clinical practice following diagnosis of COPD. Chest. 2006;129(6):1509-15. https:/ doi.org/10.1378/chest.129.6.1509

24. Nelson SB, LaVange LM, Nie Y, Walsh JW, Enright PL, Martinez FJ, et al. Questionnaires and pocket spirometers provide an alternative approach for COPD screening in the general population. Chest. 2012;142(2):358-66. https://doi.org/10.1378/chest.11-1474

25. Blumberg HM, Burman WJ, Chaisson RE, Daley $C L$, Etkind SC, Friedman LN, et al. American Thoracic Society/Centers for Disease Control and Prevention/Infectious Diseases Society of America: treatment of tuberculosis. Am J Respir Crit Care Med. 2003;167(4):603-62. https://doi.org/10.1164/rccm.167.4.603

26. Long R, Maycher B, Dhar A, Manfreda J, Hershfield E, Anthonisen N. Pulmonary tuberculosis treated with directly observed therapy: seria changes in lung structure and function. Chest. 1998;113(4):933-43. https://doi.org/10.1378/chest.113.4.933 